\title{
Test Suite Migration Experiences for B-ISUP and B-MTP
}

\author{
C. Desroches, M. Li, S. Pietsch, A. Rennoch,
} I. Schieferdecker, T. Vassiliou-Gioles GMD FOKUS

Kaiserin-Ausgusta-Allee 31, D-10589 Berlin, Germany

phone: (+49 30) 3463 7000, fax: (+49 30) 34638000 http://www.fokus.gmd.de/tip

\begin{abstract}
It is well known that the development of ATSs and ETSs is time and cost intensive. This contribution provides practical experiences and results gained from a test suite development project in the context of today's ATM protocols. In particular we discuss the problems and effort to develop and execute the BISUP and MTP test suites within B-ISDN based on corresponding N-ISDN tests. The consideration of our approach and observations should help during the production and execution of related protocol conformance tests. We have outlined basic ideas on how to underpin methodologically the ATS migration process.
\end{abstract}

Keywords

Protocol Conformance Testing, ATM, B-ISDN, BISUP, MTP, TTCN 


\section{INTRODUCTION}

The conformance assessment process defined in the Conformance Testing Methodology Framework (CTMF) standard (X.294) gives a summary of the necessary working steps for the preparation, execution and analysis w.r.t. classical protocol conformance testing. During the preparation phase questions related to testability, SUT configuration, Abstract Test Method and Abstract Test Suite (ATS) must be addressed. According to ITU-T Recommendation X.290 a protocol defining group is responsible for the Test Suite Structure and Test Purposes (TSS\&TP) documentation. The final ATS is to be produced together by protocol experts and the test realizer (see Figure 1).

Many times TSS\&TP documents are not available from the protocol defining group and the ATS is developed by testing experts with good knowledge of test cam-

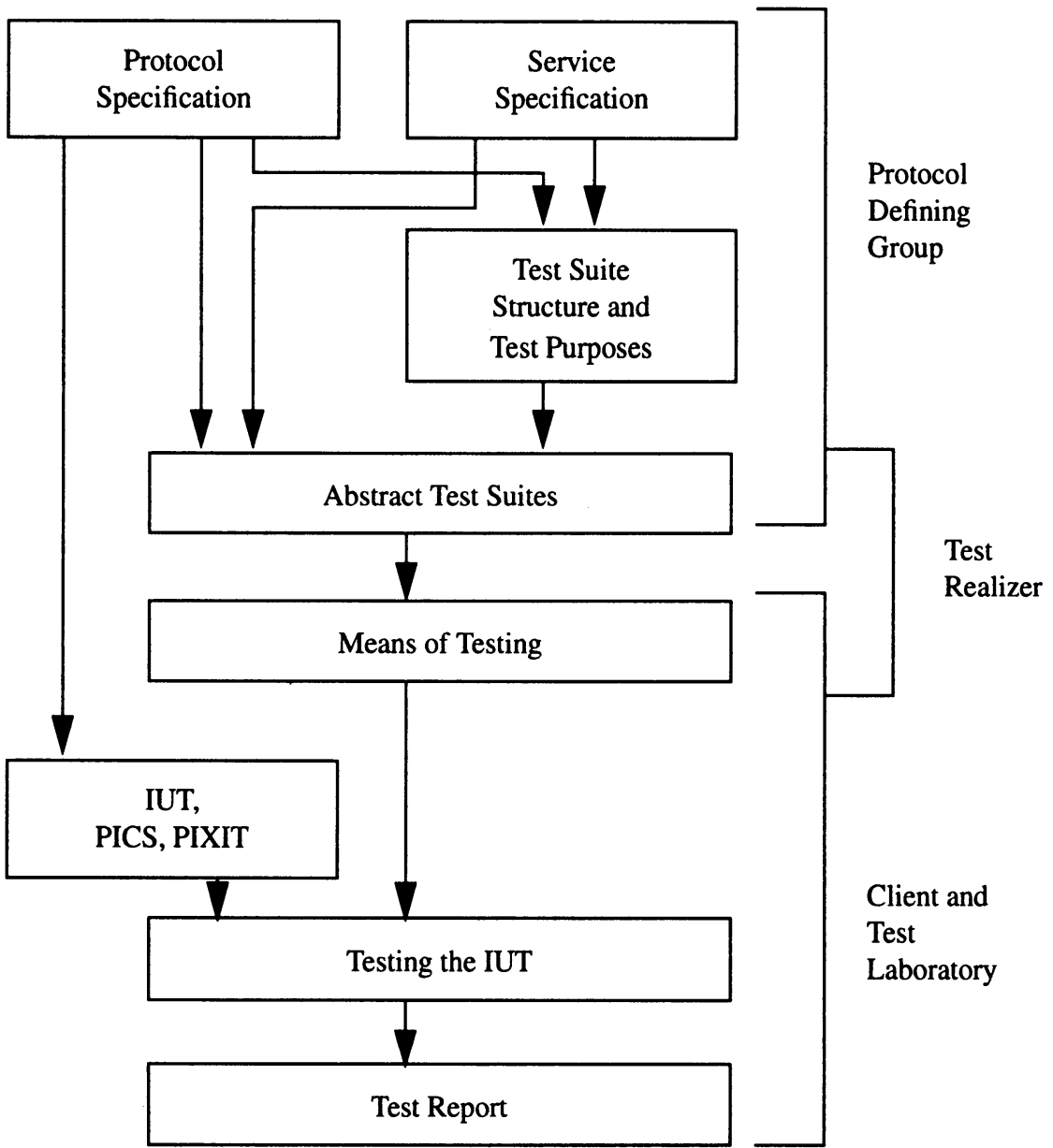

Figure 1 Relationships between parts, concepts and activities (X.290/Fig. 10). 
paign, TTCN/ASN.1 and the test equipment but having less expertise of the protocols under test.

An ideal approach to develop an ATS is the tool supported derivation of tests from a formal protocol specification. There exist a lot of experiences with the generation of TTCN tests from SDL specifications (e.g. Pérez 1997, Grabowski 1997). In this context test purposes are required to be written in Message Sequence Charts (MSC). Test case generation from SDL specification is only effective if an SDL model is available. However, to develop a SDL model might be as time consuming and labour intensive as writing a test from scratch. Time pressure will enforce a manual test suite development with the disadvantage of some incompleteness and restricted means to reason about correctness and test suite coverage.

Other approaches for test case derivation propose e.g. a step-wise knowledgebase development of test cases also starting with some test purpose formalisation (Guerrouat 1996) or even request an enhanced protocol model (König 1997). The aim in the latter proposal is to consider testability during protocol engineering to decrease the efforts in protocol testing (e.g. extra testing points should be added to observe inter-module communication).

Many of these proposals are of scientific value but do not solve the telecommunication industry work of today, i.e. development of usable ATSs in very short time frames. Unfortunately (semi-) automatic test suite derivation tools are still prototypical in nature and formal protocol specifications are often missing. Manual ATS development thus becomes a challenge in balancing sound protocol knowledge with adequate testing expertise.

In this paper we report on the approaches used and results obtained in a joint research and industry project in which abstract and executable test suites were developed within the B-ISDN / ATM framework. Several ATM Executable Test Suites (ETS) are scheduled for development. These include ETSs for the User-Network Interface (UNI) and Network-to-Network (NNI) related protocols. The scope of the project includes ATM/AAL layer as well as several ATM signalling related protocols SSCOP (Q.2110), SSCF (Q.2130, Q.2140), MTP (Q.2210), BISUP (Q.2761-64) and UNI Signalling (Q.2931 / UNI 3.1 - user and network side, Q.2961, Q.2963, Q.2971). Figure 2 gives an overview of the signalling protocol stacks for UNI and NNI nodes.

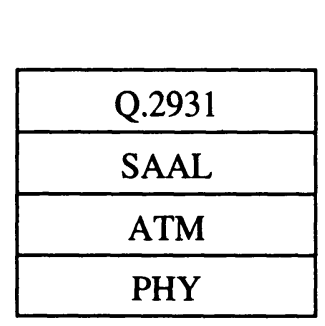

UNI

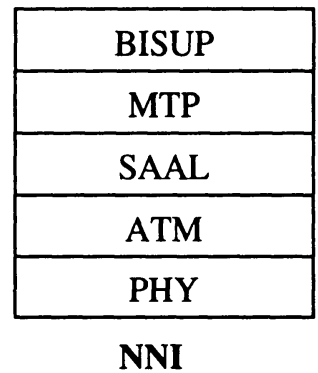

NNI

Figure 2 ATM network Signalling protocol stacks (Q.2010/Fig. 7). 
Preliminary versions of some ATSs, UNI 3.1 and AAL5, were available from the ATM-Forum. These still needed to be analysed, corrected, enhanced and/or completed and made executable. Others needed to be developed directly from the protocol specification. In this paper we focus on two examples namely BISUP and MTP.

We chose these two protocols as both have pre-existing narrowband (N-ISDN) predecessors upon which the Broadband ISDN (B-ISDN) variants are based.

Further test purpose lists and even TTCN ATSs were available for the Narrowband protocol variants. Test purpose lists and the narrowband ISUP (BISUP predecessor) ATS have been standardized by the ITU-T. The narrowband MTP ATS has been specified by ETSI. Due to this prerequisites, we will report on our approaches to consider this test purposes lists and to migrate the narrowband ATS to an enhanced B-ISDN version.

It is our aim to demonstrate how existing test suites can be migrated towards a newer technology and to show different approaches during the test suite design phase. We also provide some insight on problems and dependencies to be considered during the adaptation of the ATS to a specific System under Test and a Means of Testing (MOT).

The Tektronix K1297 TTCN development tool kit (based on Forth / VxWorks) has been selected as the protocol conformance testing platform for Executable Test Suite (ETS) development.

\section{TEST SUITE DESIGN}

The test suite development that is described below was constraint by the following side conditions:

- For each test suite, three approaches used to derive Test Suite Structures (TSS) were considered. The state based approach which defines test groups for each stable/testable protocol state. The procedure based approach which defines test groups for each protocol procedure and lastly the PDU format based approach, which defines test groups based on PDU formats.

It has been investigated which test suite structure fits best to the protocol description in the standard and to what extent the test suite structure of the narrowband test suite can be adapted to the broadband version.

- The basic principle for the development of test cases is that one test purpose is respected by exactly one test case, so that there is a one-to-one mapping between the test purpose list and the dynamic $t$ shaviour part of the test suite.

- Since in general, no interface at the upper tester PCO can be assumed, we treat the upper tester send events as corresponding implicit send events and do not include receiving event at the upper test PCO. The remote testing method is practically the most widely applicable test method. Therefore, we assume test operator involvement for test execution.

- Being a joint research and industry project, a transition in our thinking from an academic viewpoint towards an industry oriented viewpoint was in order. The ATSs could only use control structures and language features currently sup- 
ported by the TTCN compiler. Further, the runtime environment and hardware used by Tektronix needed to be taken into consideration.

\section{BROADBAND ISDN USER PART}

\subsection{Prerequisites}

The B-ISDN user part (BISUP) protocol is part of the B-ISDN Signalling protocol stack at NNI nodes (Q.2010). It realises the signalling capabilities and functions required to support basic bearer services and supplementary services for capability set $1 \mathrm{~B}-I S D N$ applications. The BISUP is applicable to international B-ISDN networks and is defined by ITU-T Recommendations Q.2761 - Q.2764. Since at transit nodes BISUP has to support the ISDN user part of Signalling System No. 7 ((N)-ISUP) services there exists a strong relationship to the corresponding narrowband ITU-T Recommendations Q.761 - Q.764.

Basic functionality of BISUP refers to the initiation and release of signalling calls due to incoming messages carrying partly complete or complete call address information. B-ISUP protocol entities must accept / confirm or reject calls based on the delivered parameters and further message exchanges with adjacent network nodes. Additional functions address the blocking or reset of signalling calls.

The data type definitions of BISUP messages and parameters are available in ASN.1 [Q.2763]. The procedural specification of BISUP given in Q.2764 is subdivided into five process functions to allow for a separation of different concerns. Consequently a large number of internal coordination signals have been defined requiring an in-depth study to understand the model specification. The related SDL diagrams are incomplete (in particular due to the data part) and are therefore not suitable for use with any (semi-)automated test case generation tool. As a consequence, the entire dynamic part, including all constraints of the test suite, were implemented manually. In protocols where complex message structures exist, development of the constraints is sometimes more labour intensive as developing the test sequences.

A starting point of the ATS development is given by the existence of an incomplete test suite of the N-ISUP protocol: In Q.784 the ITU-T gives an (N)-ISUP basic call test specification which provides a test suite structure and test purpose list. For each test purpose pre-test conditions and expected message sequences have been included. Annex A of Q.784 contains a TTCN version of Q.784. The absence of test constraints and the very intensive usage of implementation dependent upper tester primitives gives reason to believe that this TTCN test suite has never been successfully executed.

\subsection{ATS development}

The close relationship between BISUP and NISUP led us to adopt the NISUP test purposes for the BISUP test suite. First we have distinguished between:

- test cases which are not applicable to the BISUP since NISUP messages do not have any equivalent in BISUP, and 
- test cases which are still of interest in the BISUP context.

In the latter group, we updated the PDU message types and added required message exchange sequences which do not appear in NISUP (here e.g. IAA/IAR, RAM messages). A major part of the work consists in the specification of the message constraints since none of them were available. The ASN.1 message data type definition used from Q.2763 are highly substructured and therefore requires complex data constraint definitions. Sometimes it appears that the TTCN data definitions would be preferred if we would have a decision between both specifications. But in our case the ASN.1 specification was the only one available. During the analysis of message types a number of simple changes were necessary:

- The ordering of data type definitions had to be changed since the TTCN compiler did not support forward referencing and requires the parameter type definition before they could be referenced first (Q.2763 starts with the message type list).

- Many constants (e.g. "digit0") had been defined multiple times (for different parameter types). Since this causes compiler errors we have made them unique by introducing prefix identifiers (e.g. "x_digit0").

Writing a test suite always requires an early knowledge of the potential test execution configuration. We decided to keep an upper tester PCO beside the lower tester PCO to allow test cases which include IUT initiated protocol procedures. Since no interface at the upper tester PCO could be assumed we treated the upper tester send events as corresponding implicit send events and removed the receiving event at the upper tester PCO, i.e. we assume a test operator involvement. On the other side an additional lower tester PCO for the supervision of N-ISDN circuit events (e.g. check of ringing tone) become superfluous. The final basic abstract test configuration is illustrated in Figure 3.

The nature of BISUP do not require concurrent TTCN for the implementation of its test cases.

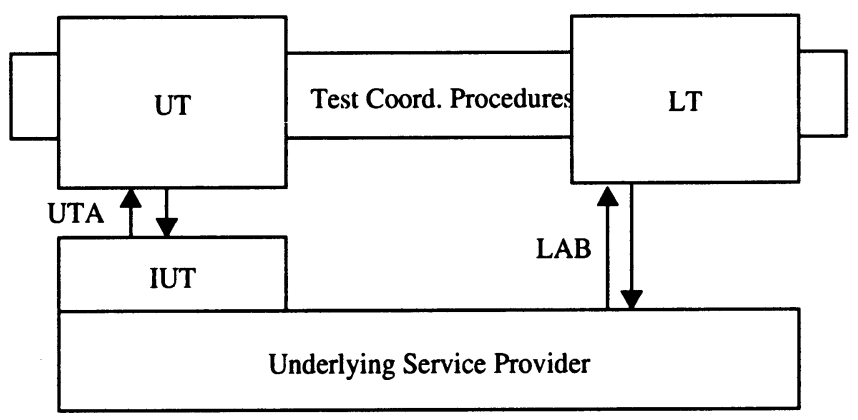

UT Upper Tester

LT Lower Tester

IUT Implementation under test

UTA Upper Tester $P C O$ at signalling point A

LAB Lower Tester PCO between service provider and signalling point $B$

Figure 3 BISUP ATS configuration. 


\subsection{Test Execution}

We derived 62 test cases (out of 75 tests) from the NISUP test suite. Although all of them use the same test configuration, special preparations for the IUT and tester configuration were required for those test cases, which include connection establishment / release initiated by the IUT. Since our IUT allows no manual message sending we installed a test configuration involving a network user at an UNI interface of the IUT (see Figure 4). The user behaviour at the UNI is driven by the test equipment.

This configuration is compatible with the abstract test configuration of the BISUP ATS. The test operator engagement (which is specified in the ATS via implicit send events at the UT PCO) will be realised with appropriate signalling call requests at the UNI interface UTA (using messages of ITU-T recommendation Q.2931). E.g. the establishment of an UNI signalling connection (SETUP message calling the "network node LT") leads the IUT to issue an IAM (BISUP Initial Address message) at LAB. It is clear that the IUT had to be configurated with appropriate calling address numbers of the UT "user" and the LT "network node". We had to note that this configuration is not specified within the ATS and has been selected due to the available IUT properties only.

The test suite has been compiled and executed with the Tektronix K1297 protocol tester and the Siemens EWSXpress ATM switch was used as IUT. 45 test cases were executed successfully, the remaining test cases could not be performed completely due to restrictions with the IUT. In particular, a number of IUT procedures could not been started with the upper tester configuration.

After installing a suitable basic test configuration we will extend the list of test purposes to further tests with messages of minor concern (e.g. User Part test message) and B-ISDN specific protocol procedure as consistency check. Also special test on the interworking between BISDN and ISDN are envisaged.

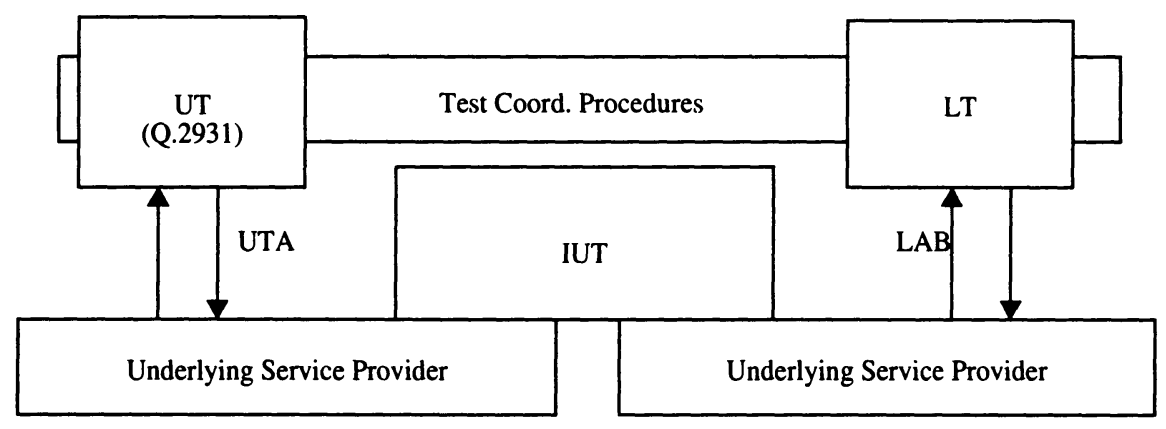

UT Upper Tester

LT Lower Tester

IUT Implementation under test

UTA Upper Tester PCO at signalling point A

LAB Lower Tester PCO between service provider and signalling point B

Figure 4 BISUP test experiment configuration. 


\section{BROADBAND ISDN MESSAGE TRANSFER PART}

\subsection{Prerequisites}

The B-ISDN Message Transfer Part Layer 3 (MTP3b) protocol is part of the ITU-T Recommendation Q. series on switching and signalling network protocols. It defines the protocol for connectionless transfer of signalling messages between signalling points (SPs) which are nodes in a Common Channel Signalling network. MTP3b is sandwiched between SAAL (SSCF network side and SSCOP) below and one of various user parts (BISUP, TUP, SCCP and TCAP) above (see e.g. Figure 2).

MTP3b defines the functions and procedures required for signalling message handling and signalling network management. Signalling message handling is responsible for message discrimination where the Signalling Point (SP) determines whether a message is for itself or for another SP. If it is for itself, the message is forwarded to the message distribution function which then determines the appropriate User Part to which a message should be sent. If the message is not for itself, then it is forwarded to its message routing function which forwards messages onto the appropriate link(s) or discards the message if it cannot determine the proper receiver.

Network management is used for network reconfiguration in case of link or SP failures as well as for traffic flow control in case of network congestion. Predetermined routing information is required to achieve this.

MTP3b is specified in ITU-T Recommendation Q.2210. Q.2210 itself is derived from ITU-T Recommendations Q.704 and Q.707, which define the functions and procedures for Narrowband MTP (N-MTP). MTP3b message formats are very similar to those in N-MTP. Major differences in message formats are limited to the extension of the maximum size of a Service Data Unit due to the capabilities of the underlying protocol layer ( 272 octets within narrowband and 4096 octets in broadband). Further the changeover message has been extended slightly.

We re-used existing N-MTP layer 3 testing information. ITU-T Recommendation Q.782 defines the Narrowband MTP layer 3 test specification. Further an ETSI MTP3b ATS based on Q.782 exists. It uses the same top level test group structure as in Q.782 with the exception that each top level test group is further subdivided into combinations of valid, invalid and inopportune groups.

Q.782 defines a set of tests and four test configurations for testing an SS\#7 Narrowband MTP layer 3. Which configuration applies to the test and what type of signalling point (switch) applies to the test, the type of test, validation or capability, is provided. However, the test case bodies are described using an arrow notation accompanied with comments (see Figure 5 for details).

We adopted Q.782 as a starting point in the development of the MTP3b ATS since it was already an established standard, and it provided a comprehensive list of test purposes, as well as detailed testing configurations for testing SPs. 


\subsection{Test Suite Structure and Test Purposes}

Since both Q.782 and the ETSI Narrowband MTP ATS use a procedure based approach to define the ATS TSS, and since the MTP3b ATS is also based on Q.782, it was reasonable to use the same approach for defining the MTP3b TSS.

The ETSI ATS subdivides each procedure test group into valid, invalid and inopportune groups. The MTP3b ATS in comparison has no further subgrouping. Only valid test cases are provided. A separate test group containing test cases for invalid behaviour is provided.

Many of the test purposes in Q.782 were rewritten. Either the expected IUT behaviour was missing entirely from the test purpose, or was provided elsewhere, usually in the Test description, or could only be determined by analysing the message sequences themselves and referring to Q.704. For example, the test purpose for Q.782/13.9 states: "To check the actions of the system on reception of an invalid traf-

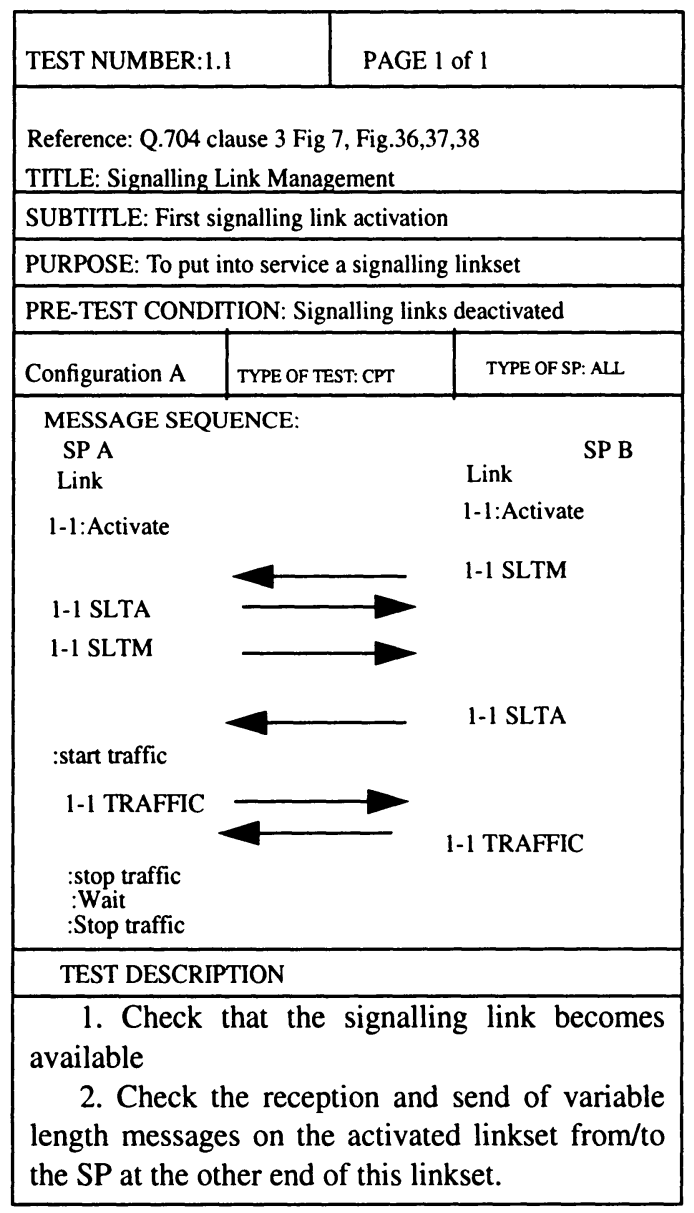

Figure 5 Sample Q.782 Test Case Description. 
fic restart allowed message". In this particular case, the expected IUT behaviour is found in the test description. The test purpose was rewritten as follows: "Verify that the IUT ignores a traffic restart allowed (TRA) with an unknown OPC)". In this way, both the input is identified and the expected output or behaviour is clearly stated.

In some cases, predominantly in the Invalid Messages, where the IUTs behaviour on receipt of invalid messages is verified, test cases were split into several test cases, sometimes generating up to 16 test cases from a single test. Since a test case will stop executing on final verdict assignment, which generally occurs on observing a nonconformant behaviour, it cannot be guaranteed that all messages will have been tested, thus causing incomplete coverage. One advantage of such test cases is that it may detect compound errors, where several erroneous messages must be sent before an error appears. The principle of one test purpose one test sequence was applied.

Q.782 describes tests when initiated from the Tester side. The comments in individual test cases require that the same test be repeated, but when initiated from the IUT side. This automatically doubles the number of test cases in the ATS. Incomplete test case descriptions required further work to determine the missing behaviour and to completely define test cases. Q.782 uses arrow diagrams. This is very descriptive and simple to understand. Fortunately MTP3b message formats are simple and derivation of PDU constraints based on these descriptions was straightforward.

The MTP3b ATS includes test cases related to the most useful configuration providing maximum protocol coverage, i.e. configuration $A$ of $Q .782$. It uses one signalling link set with one to four signalling links simultaneously. Other configurations were thought not to be implementable because of limitation in the number of PTCs allowed to execute concurrently. This is not the case. If one only takes the signalling links existing between the IUT and the tester, and assumes that all other SPs and signalling links can be simulated within the tester, it can be shown that at most 6 signalling links is required. The testing environment easily supports this requirement. The MTP3b ATS could therefore be extended to include test cases for all test configurations defined in Q.782.

\subsection{ATS Development}

The ETSI Narrowband MTP Level 3 ATS is defined using non-Concurrent TTCN. The arrow diagrams used to describe Q.782 test cases suggest that MTP3b activities are parallel in nature. Some MTP3b activities are inherently parallel in nature. E.g. MTP3b defines a load sharing procedure whereby traffic targeted for a given destination can be sent on different signalling links. How an IUT selects a signalling link and the order in which signalling links are selected to carry traffic to its destination is IUT specific. The ATS should therefore be written such that this is transparent. Further the ATS must be written in such a way that errors such as duplicate, mis-sequenced or lost messages can be detected. In a non-concurrent approach, mis-sequencing of messages can only be detected by requiring that messages be sent in a 'known' order. This places an unnecessary constraint on an implementation. Using concurrent TTCN, mis-sequencing of messages can be correctly detected without 
placing this same constraint on the IUT.

Using concurrent TTCN requires coordination between test components. ATS complexity thus increases. One of the major stumbling blocks in non-Concurrent TTCN when attempting to test a process which is concurrent in nature is the ordering of messages. One is forced to enumerate all possible sequences in which messages can arrive. This makes for unwieldy and unnecessarily complicated test cases. Especially this factor led us to conclude that the correct approach was use of concurrent TTCN although the ETSI ATS use no concurrence.

In some cases error indications must be communicated to the management layer. The MTP3b ATS ignores these as they are generally not observable in most cases, and tend to complicate test cases unnecessarily. Only observable, and sometimes indirectly observable behaviours are modelled in this ATS.

The MTP3b ATS went through an iterative process of defining the test configuration, test suite structure and test cases, to increase the test suite coverage and to make the test suite executable. The different versions are described below.

\subsubsection{First MTB3b ATS Version}

The test case structure used was intentionally very generic thus allowing for reuse with minimal change. One test case structure was designed per test configuration and depending on how many signalling links were used in the test case. This required defining highly parameterized test steps some having in excess of 6 parameters. To maintain the generic test case structure all CREATE statements were parameterized with the same test step. This test step consisted of one huge switch statement which based on an index provided as an actual parameter, would then call the appropriate test step to perform the actions associated with a given test case.

In this first approach a true master/slave relationship existed between the Main Test Component (MTC) and the Lower Tester Parallel Test Components. Intelligence was centralized in the MTC, where the MTC would send/receive PDUs to/ from the Lower Testers (LTs) encapsulated within Coordination Messages (CMs) sent via CPs.

The Upper Tester (UT) was controlled by the MTC. It was responsible for generating Test Traffic and forwarding it to the IUTs' MTP3b upper interface. Its behaviour was more or less independent from the MTC, requiring no explicit PDU exchanges with the MTC and therefore was implemented as a self standing PTC.

\subsubsection{Second MTP3b ATS Version}

Our first attempts at viewing executed test cases, simulated in this case, was very difficult, because of the high level of parameterization, the parallel nature of execution, and the level of nesting. For these reasons, excessive test step parameterization was reduced by hard coding PCOs, CPs and other parameters directly into test steps. Further, the generic test step used in CREATE statements in test cases was replaced by direct 'calls' to the appropriate test step. This reduced the ATSs nesting depth by one. These changes improved the ATS readability tremendously, both in the GR version of ATS and during test case simulation execution on the K1297. 
Further, intelligence was 'pushed down' into the LTs. As with the UT, LTs behaved independently but were nevertheless controlled by the MTC using CMs. PDU exchanges between the MTC and LTs was completely eliminated for three reasons. First, avoid the problem associated with message ordering and the sheer number of possibilities especially when several LTs executed simultaneously. Second, continuing with the same structure would make the MTC hopelessly unreadable and complicated. Lastly, the K1297 did not correctly support the use of the Meta-PDU type.

Minor changes were made to provide for more user friendly data entry. Further changes were required because of problems related to the use of forward referencing where assignment of constraints and PDUs to test case variables was not supported.

\subsubsection{MTP3b ATS Version with Changeover}

The MTP3b signalling network management (SNM) changeover procedure ensures that signalling traffic carried by an unavailable link is diverted to alternative link(s) while avoiding message loss, duplication and mis-sequencing. When a signalling link fails, transmission and reception of messages on the failed link is terminated, alternative link(s) are selected, the faulty link's retransmission buffer is updated and traffic is diverted to the identified alternative signalling link(s).

Buffer updating consists of transferring the concerned messages to the alternative link(s) retransmission buffer(s). Signalling links in the ATS are modelled as PCOs. Unsent messages must first be retrieved from the inactive link buffers, transferred from one PTC to another via CMs, copied field by field to another PDU sent through the PCO designated as the alternative link.

With no direct PTC to PTC communication permitted, this entailed unnecessary communication, data copying and unnecessary processing overhead. For this reason, the model was modified to permit direct PTC to PTC bidirectional communication via special CPs (LCP1_2 etc.) (see Figure 6).

The alternative link must handle traffic from the now deactivated link, its own traffic, and transmit unsent traffic from the deactivated link. Even with this new PTC to PTC communication possible, it was feared that run time performance of change-

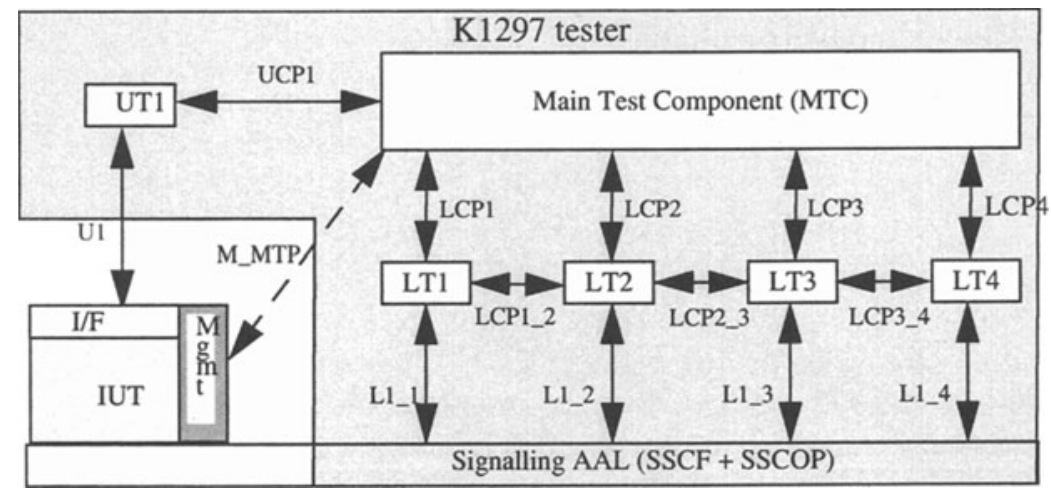

Figure 6 MTP3b Test configuration for a SEP. 
over would be inadequate. The TTCN code responsible for transferring unsent data from one signalling link's buffers to the alternative signalling links buffers for retransmission was therefore re-implemented using a test suite operation, where transparent communication between signalling links and their respective transmission buffers took place.

\subsubsection{MTP3b ATS Version with Test Traffic Generation}

Two testing models are defined for generating test traffic in Q.782. The first, test traffic is generated by the upper tester and delivered to the IUT via MTP Transfer request primitives. Test traffic received by the lower testers is reflected back to the IUT and should in theory be received by the upper tester to identify any loss of the test traffic.

If an IUT does not support an adequate MTP3b Upper Boundary interface, and does not provide any endpoint functionality, the ATS configuration must be changed according to Figure 7: Instead of the UT we introduce another lower tester (GEN) which takes over the test traffic generation task of the UT. Now the MTP messages are sent from a LT test component to the IUT which forwards the traffic to one of the components LT1 - LT4.

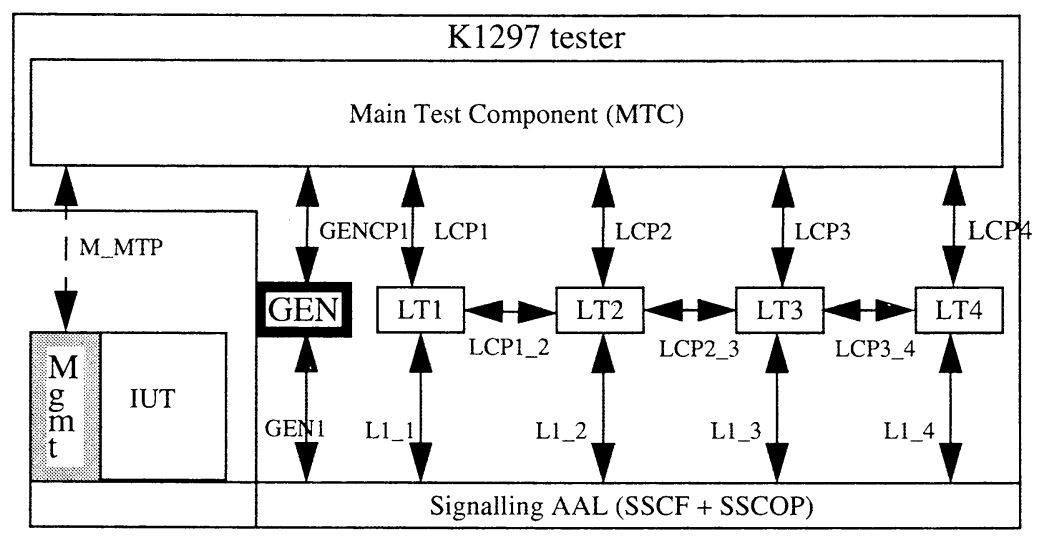

Figure 7 MTP3b Test configuration for a STP.

\subsection{Test Execution}

Q.782 contains 87 test case descriptions for Configuration A. The MTP3b ATS defines 204 test purposes, of which 94 are currently implemented in TTCN.

The ATS was verified using the Tektronix K1297 TTCN compiler and executable code was produced. Currently, our means of testing does not support the functionality required to perform changeover procedures and some of the ATS defined ASPs were not yet supported by the underlying SAAL implementation, so that the ETS can be used only in a restricted manner. However, the next release of the means of testing will cope with these problems. 
One major stumbling block remained, namely, the unavailability of an IUT against which we could further verify our ATS. Three alternatives were considered: writing a complete set of MTP3b simulation scripts for our purposes, writing or purchasing an MTP3b emulation or lastly finding an existing MTP3b implementation, public domain if at all possible for cost reasons.

The effort required to write a complete set of simulation scripts or write our own emulation to verify our ATS was deemed to be too high. Therefore using various search engines we identified companies having MTP3b implementations. Recently, we established a joint project with a company which will provide us with a MTP3b implementation. In return they will receive a complimentary conformance test report.

\section{CONCLUSIONS}

The discussion on the migration of Narrowband ISDN to the Broadband ISDN technology environment has shown the various aspects of test suite development for today's ATM protocols (here BISUP and MTP). In both examples we have similar prerequisites: available TSS\&TP documentation, some more or less standardized and useful ATS for N-ISDN and the same (migration) target. But due to the nature of these two protocols our experiences and efforts with this work have been very different. In particular, the introduction of parallel behaviour and the use of concurrent TTCN in the MTP ATS causes a big expense to make the final ATS design and implementation.

Due to our experiences and project results we have started research in the following directions: One aspect is that the usage of Concurrent TTCN in large and complex test suites lead us to think about some tool support during the development of ATS including concurrence. At GMD Fokus a test suite simulator (Pietsch 1998) has been implemented for verification of dynamic aspects and validation of logical correctness of concurrent TTCN test suites. The simulator has been developed using the ITEX C-Code Generator CCG for the interpretation and execution of TTCN code.

Further, from the ATS migration project arises the question of test suite generation from another viewpoint. Here we did not try to derive an ATS from a formal protocol description, but we focused on the enhancement of existing ATSs to an updated protocol standard. The question is about some test suite structure and abstract test case dynamic behaviour sequences which make an ATS suitable for periodical protocol (version) updates.

Furthermore, we see test cases related to classes of viewpoints like protocol state transitions (e.g. timeouts), procedure (e.g. data correction methods) or message format verification (PDU parameter field contents) and ask for a generic test suite structure to be adopted to different protocols and instantiated to become a helpful starting point in the ATS development process. Such documents shall serve as a constructive working platform for new ATSs and are a must to go beyond general methodology guidelines or naming conventions in the ATS production process. We think of test suite specification guidelines which are similar to specification guidelines adopted 
for LOTOS (Bolognesi 1995). Another area of research is to apply the framework idea known from object-oriented software development process to the area of test suite development (and migration in particular).

\section{ACKNOWLEDGEMENTS}

We would like to thank Tektronix Berlin GmbH, in particular Jens Kittan and Wolfgang Borgert, who supported the compilation and usage of the test suites with the K1297 protocol tester.

\section{REFERENCES}

Bolognesi, T., v.d.Lagemaat, J., Vissers, C. (1995): LOTOSphere: Software Development with LOTOS. Kluwer Academic Publishers.

Guerrouat, A., König, H. (1996): Automation of test case derivation in respect to test purposes. In: ICTCS'96 proceedings. Darmstadt (Germany).

Grabowski, J., Scheurer. R., Dai, Z.R., Hogrefe, D. (1997): Applying SAMSTAG to the B-ISDN protocol SSCOP. In: ICTCS' 97 proceedings. Cheju Island (Korea).

ITU-T Recommendation Q.704 (1993) Messages Transfer Part. Signalling Network functions and messages.

ITU-T Recommendation Q.707 (1993) Specifications of signalling system No. 7, Testing and maintenance.

ITU-T Recommendation Q.761 - Q.764. Specification of Signalling System No. 7.

ITU-T Recommendation Q.782 (1993) Specification of signalling system No. 7. Signalling system No. 7 - MTP Level 3 test specification.

ITU-T Recommendation Q.784 (1991). ISUP Basic Call Test Specification.

ITU-T Recommendation Q.2010 (1995). Broadband Integrated Services Digital Network Overview - Signalling Capability Set 1, Release 1.

IUT-T Recommendation Q.2110. B-ISDN ATM Adaptation Layer-Service Specific Connection Oriented Protocol (SSCOP).

ITU-T Recommendation Q.2130 (1995) B-ISDN ATM Adaptation layer - service specific coordination function for signalling at the user network interface.

ITU-T Recommendation Q.2140 (1995) B-ISDN ATM Adaptation layer - service specific coordination function for signalling at the network node interface.

ITU-T Q.2210 (1995) Messages transfer part level 3 functions and messages using the services of Q.2140.

ITU-T Recommendation Q.2761 - Q.2764 (1995). B-ISDN User Part (B-ISUP) of Signalling System No. 7.

ITU-T Recommendation Q.2931 (1995). B-ISDN User-Network Interface (UNI), Layer 3 Specification for Basic Call/Connection control.

ITU-T Recommendation Q.2961 (1995). B-ISDN - Digital subscriber signalling system no.2 (DSS 2) - Additional traffic parameters.

ITU-T Recommendation Q.2963 (1996). DSS 2 - Connection modification.

ITU-T Recommendation Q.2971 (1995). UNI layer 3 specification for point-to- 
multipoint call/connection control.

ITU-T Recommendation X.290 (1991). OSI Conformance Testing Methodology and Framework (CTMF) - Part 1: General Concept.

ITU-T Recommendation X.292 (1993). OSI CTMF - Part 3: The Tree and Tabular Combined Notation (TTCN).

ITU-T Recommendation X.294 (1991). OSI CTMF - Part 4: Test Realization.

König, H., Ulrich, A., Heiner, M. (1997): Design for testability: a step-wise approach

to protocol testing. In: ICTCS'97 proceedings. Cheju Island (Korea).

Pérez, E., Algaba, E., Monedero, M. (1997): A pragmatic approach to test generation. In: ICTCS' 97 proceedings. Cheju Island (Korea).

Pietsch, S., Schieferdecker, I. (1998): Test Suite Simulation. In: FBT'98 proceedings. Shaker Verlag, Germany.

Tektronix Protocol Test System K1297. User Manual (1996).

\section{BIOGRAPHY}

Claude Desroches holds an Honours BSc. in Computer Sciences from the University of Ottawa in Canada. He has worked as a software engineer in the field of telecommunications since 1987 with Bell Canada, the University of Ottawa, HewlettPackard/Idacom and Tekelec Inc. His research interests include Formal Description Techniques, compiler development, implementation, validation, verification, and conformance testing of protocols. He is currently a guest researcher at GMD Fokus.

Mang Li has been studying at the Institute of Telecommunication Networks, department of electrical engineering, Technical University Berlin. She has been working as a student at GMD Fokus since 1996. She is interested in conformance testing and performance testing.

Stephan Pietsch studied electrical engineering at the Technical University Berlin. He is a scientist at GMD Fokus in Berlin. His research interests are in conformance and interoperability testing of communication protocols.

Axel Rennoch studied mathematics at the Free University of Berlin. His research interests include the application of Formal Description Techniques for testing methodologies and Quality of Service considerations. Currently he is employed as a scientist at GMD Fokus in Berlin.

Ina Schieferdecker studied mathematical computer science at the Humboldt University in Berlin and received her Ph.D. from the Technical University in Berlin in 1994. She attended the postgraduate course on open communication systems at the Technical University in Berlin. Since 1993, she is a researcher at GMD Fokus and a lecturer at Technical University Berlin since 1995. She is working on testing methods for network components, and carries out research on formal methods, performance-enhanced specifications and performance analysis.

Theofanis Vassiliou-Gioles studies electrical engineering at the Technical University Berlin. His main fields of research interests include protocol validation, conformance and interoperability testing and distributed testing methodologies. Currently he is employed at GMD Fokus. 